\title{
REDUÇÃO DO CONSUMO DE OXIGÊNIO EM UM ALTO-FORNO A PARTIR DO AUMENTO DA EFICIÊNCIA ENERGÉTICA DOS AQUECEDORES DE AR
}

Charles Sóstenes Assunção I

\section{Resumo}

Este trabalho relata o estudo de melhoria de desempenho dos trocadores de calor do tipo Glendon da V\&M do Brasil. O objetivo é reduzir o consumo de oxigênio utilizado para enriquecer o ar que é soprado no Alto-Forno 2 a partir do aumento da eficiência energética dos trocadores de calor. Um modelo computacional em CFD (Computer Fluids Dynamics) foi desenvolvido para simular o comportamento dos fluidos nos Glendons. A partir deste modelo, foram simuladas algumas alterações geométricas no equipamento fazendo com que os gases da combustão escoassem de maneira cruzada em relação ao fluxo de ar. A simulação demonstrou um potencial de aumento de eficiência energética de $15,73 \%$, o que representaria um aumento de temperatura de saída do ar de $115^{\circ} \mathrm{C}$. As alterações simuladas foram implantadas em um dos três trocadores de calor que, juntamente com melhorias no controle do processo de combustão, geraram um aumento real de temperatura de $135^{\circ} \mathrm{C}$. Após a implantação destas alterações nos outros Glendons, a redução esperada do consumo de oxigênio de enriquecimento do ar no Alto-Forno 2 é de aproximadamente $4.400 .000 \mathrm{Nm}^{3}$ por ano.

Palavras-chave: Trocador de calor; Eficiência energética; Consumo de oxigênio.

\section{REDUCTION OF OXYGEN CONSUMPTION IN THE V\&M DO BRASIL'S BLAST FURNACE 2 THROUGH THE IMPROVEMENT OF THE HEAT EXCHANGERS}

\begin{abstract}
This work concerns the performance improvement study of the Glendon heat exchangers of the V\&M do Brasil. The objective is to reduce the consumption of the oxygen used to enrich the blown air in the Blast Furnace 2 through the improvement of the energetic efficiency of the heat exchangers. A CFD (Computer Fluids Dynamics) model was developed in order to simulate the behavior of the fluids inside the heat exchangers. Some geometric alterations were simulated in the model, which objective was to change the parallel flow of the combustion gases to a cross flow. The simulation showed a potential of energetic efficiency improvement of $15,73 \%$, which would mean an increase of $115^{\circ} \mathrm{C}$ in the outlet air temperature. The simulated alterations were implemented in one of three heat exchangers and the actual temperature increase was $135^{\circ} \mathrm{C}$. After the implementation of the alterations in the others heat exchangers, the expected reduction of the oxygen consumption in the Blast Furnace 2 is about $4.400 .000 \mathrm{Nm}^{3}$ per year.
\end{abstract}

Key words: Heat exchanger; Energetic efficiency; Oxygen consumption.

\section{INTRODUÇÃO}

A V\&M do Brasil S.A., situada em Belo horizonte, tem produção anual de aço em torno de 670 mil toneladas. Seus principais produtos são tubos de aço sem costura, especialmente voltados para a indústria petrolífera, a indústria automotiva e a indústria mecânica.

Para a obtenção do ferro gusa, matéria-prima na produção do aço, a V\&M do Brasil possui dois altos-fornos a carvão vegetal, que juntos são capazes de produzir 1.900 toneladas por dia. processo nos altos-fornos requer determinados valores de temperatura de chama de combustão para que a redução do minério ocorra de maneira adequada. ${ }^{(1,2)} \mathrm{De}$ modo geral, a temperatura de chama em um processo de combustão com um mesmo combustível é diretamente proporcional a pelo menos duas variáveis, ${ }^{(3,4)}$ a concentração de oxigênio no ar de combustão e a temperatura do ar de combustão, conforme mostra a Figura I.

Quanto maior for a concentração de oxigênio no ar de sopro, maior será a temperatura de chama. Contudo, o enriquecimento do ar de sopro com oxigênio é uma

\footnotetext{
' Engenheiro.V\&M do Brasil S.A, Av. Olinto Meireles, 65, Barreiro, Cep 30640-010, Belo Horizonte, MG, Brasil. E-mail: charles.assuncao@vmtubes.com.br
} 
prática indesejável, pois implica no aumento dos custos de produção. Por outro lado, a temperatura de chama pode ser elevada a partir do aumento da temperatura do ar de combustão.

$\mathrm{O}$ ar soprado no alto-forno 2 da V\&M do Brasil S.A é aquecido em três aquecedores do tipo Glendon. Esses aquecedores possuem um regime de trabalho contínuo e consistem em câmaras de paredes refratárias, onde ocorre a combustão do gás de alto-forno recuperado. $O$ calor gerado pela combustão aquece $o$ ar que passa em tubos de aço, chamados de serpentinas, conforme mostrado na Figura 2.

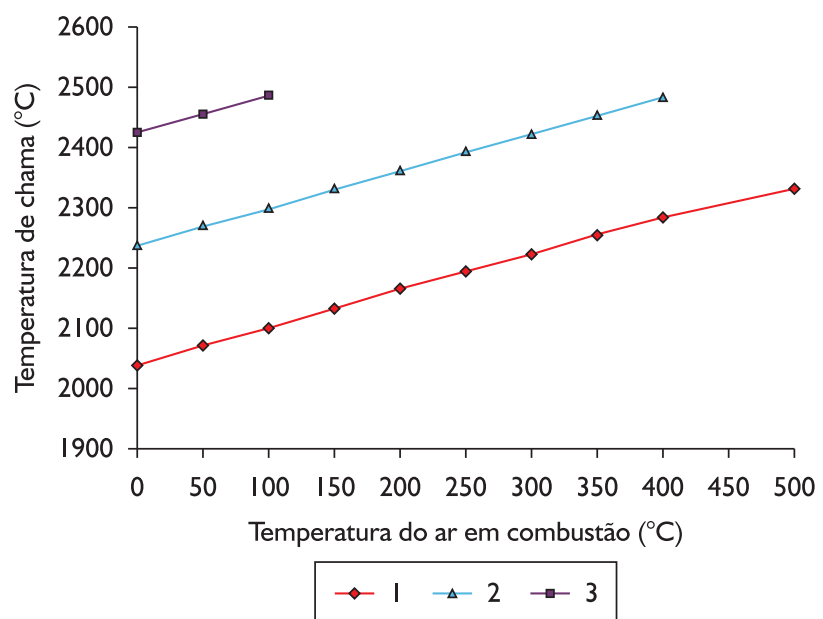

Figura I. Relação da temperatura de chama do gás natural com a temperatura de sopro e concentração de oxigênio. (linha I: $21 \%$ de $\mathrm{O}_{2}$, linha 2: $24 \%$ de $\mathrm{O}_{2}$, linha 3: $27 \%$ de $\left.\mathrm{O}_{2}\right) \cdot{ }^{(5)}$

Atualmente, o ar de sopro do alto-forno 2 é enriquecido com oxigênio em $4 \%$ do seu volume, pois a troca de energia nos Glendons não é suficiente para elevar a temperatura do ar de sopro a valores requeridos pelo processo, pois a eficiência energética dos Glendons é da ordem $59,1 \% .^{(7)}$

Este estudo foi desenvolvido com os objetivos de aumentar a eficiência dos Glendons, e conseqüentemente aumentar a temperatura do ar de saída e adequar a temperatura da câmara de combustão. Um modelo dos Glendons foi desenvolvido em CFD (Computer Fluids Dynamics) e algumas modificações geométricas foram simuladas. Com base nos resultados, foi possível identificar alternativas para que os objetivos fossem alcançados.

\section{MATERIAIS E MÉTODOS}

Este estudo foi dividido em três etapas, e em todas elas as mesmas condições operacionais foram consideradas.

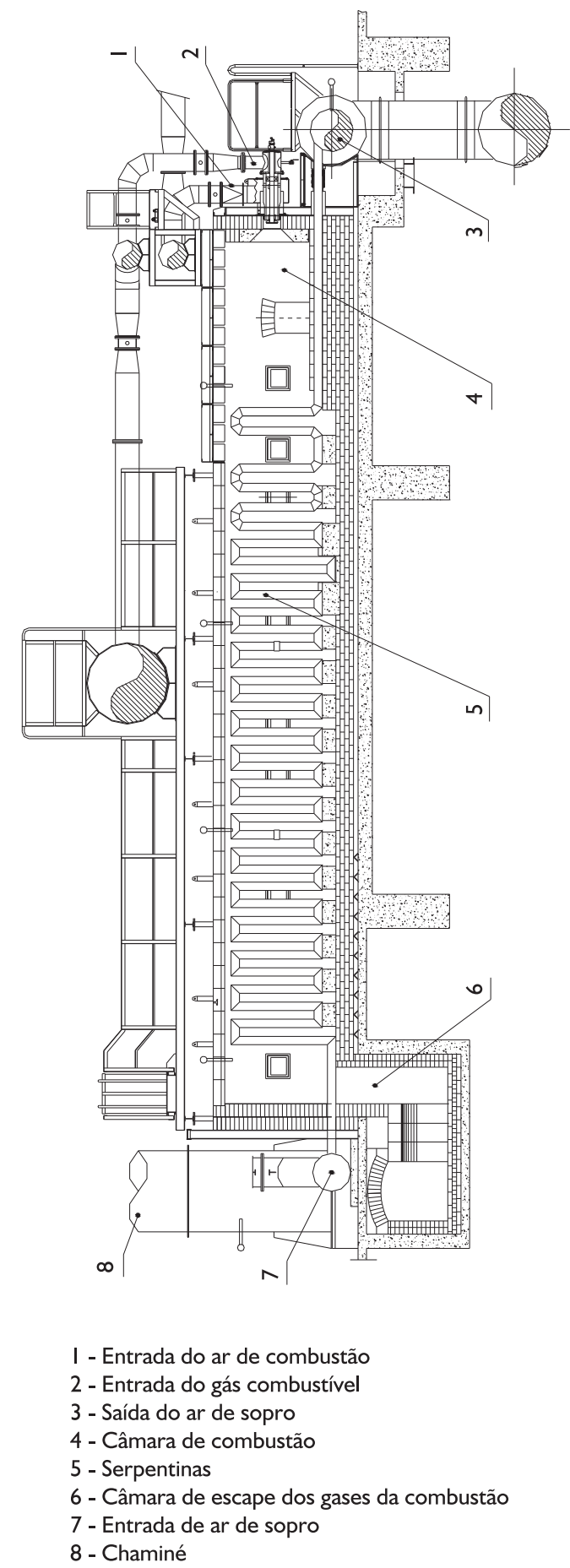

Figura 2. Corte longitudinal do Glendon. ${ }^{(6)}$

A primeira etapa consistiu em construir um modelo do comportamento fluidodinâmico das condições atuais em CFD (Computer Fluids Dynamics) e resolver as que governam o escoamento de fluidos Newtonianos, ${ }^{(8)}$ conforme mostrado a seguir.

- Conservação da massa para regime permanente

$\operatorname{div}(\rho \mathrm{u})=0$ 
- Conservação da quantidade de movimentos nos eixos $x, y$ e $z$

$\operatorname{div}(\rho u u)=-\frac{\partial p}{\partial x}+\operatorname{div}(\mu \operatorname{gradu})$

$\operatorname{div}(\rho v u)=-\frac{\partial p}{\partial y}+\operatorname{div}(\mu \operatorname{gradv})$

$\operatorname{div}(\rho w u)=-\frac{\partial p}{\partial z}+\operatorname{div}(\mu \operatorname{grad} w)$

- Equação da energia interna

$\operatorname{div}(\rho i u)=-\operatorname{pdiv}(u)+\operatorname{div}(\operatorname{kgrad} T)+\Phi$

$$
\Phi=\mu\left\{\begin{array}{l}
2\left[\left(\frac{\partial u}{\partial x}\right)^{2}+\left(\frac{\partial v}{\partial y}\right)^{2}+\left(\frac{\partial w}{\partial z}\right)^{2}\right]+ \\
+\left(\frac{\partial u}{\partial y}+\frac{\partial v}{\partial x}\right)^{2}+\left(\frac{\partial u}{\partial z}+\frac{\partial w}{\partial x}\right)^{2}+ \\
+\left(\frac{\partial v}{\partial z}+\frac{\partial w}{\partial y}\right)^{2}
\end{array}\right\}+\lambda(\operatorname{divu})^{2}
$$

- Equação de estado, considerando o ar como gás ideal

$$
\begin{aligned}
& p=\rho R T \\
& i=C_{v} T
\end{aligned}
$$

Sendo as propriedades do ar: $\rho$ a massa específica, $\mu$ a viscosidade dinâmica, $\mathrm{k}$ a condutividade térmica, $\mathrm{T}$ a temperatura, $\mathrm{p}$ a pressão, $\mathrm{u}$ a velocidade total, $\mathrm{R}$ constante, i energia interna, $C_{v}$ calor específico

Os modelos geométricos foram gerados no aplicativo Gambit a partir de desenhos de projeto dos Glendons, e as malhas foram geradas no aplicativo FLUENT. Nesta análise, algumas hipóteses foram adotadas:

- regime do escoamento: estacionário, compressível e turbulento;

- modelo de turbulência: K-epsilon;

- modelo de radiação: ordenadas discretas;

- fluido de trabalho: ar (dentro e fora das serpentinas) tratado como um gás ideal;

- coeficiente de absorção do ar (dependência linear da temperatura, em $\mathrm{I} / \mathrm{m}$ ): 0 a $95^{\circ} \mathrm{C}$ e 0,2 a $1.100^{\circ} \mathrm{C}$

- emissividades: 0,8 (aço) e I (refratários);

- temperatura do ar na entrada das serpentinas: $95^{\circ} \mathrm{C}$;

- pressão do ar na entrada das serpentinas: I, 12 kgf $/ \mathrm{cm}^{2}$;

- vazão do ar no interior das serpentinas: $9.969,3 \mathrm{~m}^{3} / \mathrm{h}$;

- temperatura do ar nos queimadores: $1.033^{\circ} \mathrm{C}$;

- pressão do ar nos queimadores: I kgf/cm²;

- vazão do ar nos queimadores: $10.063 \mathrm{~m}^{3} / \mathrm{h}$;
- queimadores: a combustão não foi modelada diretamente. Adotou-se a entrada de ar com uma temperatura elevada que permitisse introduzir no modelo uma energia equivalente à energia injetada pelos queimadores; ${ }^{(9)}$

- material das serpentinas: aço padrão do Fluent;

- material das paredes dos Glendons: refratários (paredes e isoladas);

A segunda etapa consistiu em simular a redução da área de passagem dos gases da combustão na seção transversal do Glendon, com o objetivo de aumentar sua velocidade e melhorar sua distribuição. $O$ teto foi rebaixado em $100 \mathrm{~mm}$ e cada parede lateral foi aproximada das serpentinas em $50 \mathrm{~mm}$.

A terceira etapa incluiu as alterações simuladas na etapa anterior e incluiu a introdução de cinco barreiras, que bloqueiam dois terços da seção transversal dos Glendons, funcionando como chicanas, conforme mostrado na Figura 3. O objetivo foi fazer com que o fluxo dos gases da combustão fosse cruzado ao fluxo de ar no interior das serpentinas e a turbulência do escoamento externo fosse aumentada, aumentando assim a troca de calor.

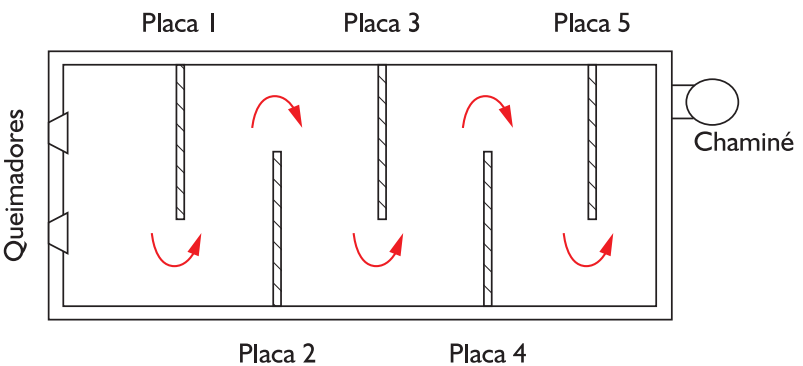

Figura 3. Placas para alteração do fluxo dos gases de combustão (adaptado). ${ }^{(10)}$

\section{RESULTADOS E DISCUSSÃO}

Para validar o modelo computacional, foram feitas medições de temperatura do ar na entrada e na saída do Glendon. As temperaturas dos gases da combustão foram medidas em 12 posições e a três alturas em cada posição (as letras A, B e C referem-se à altura do instrumento em cada posição), totalizando 36 pontos de medição, conforme mostrado nas Figuras 4 e 5.

As temperaturas obtidas pelo modelo foram comparadas com as temperaturas obtidas experimentalmente, e o erro médio entre estas temperaturas é de $16 \%$. No caso da temperatura de saída do ar, o valor medido foi $734^{\circ} \mathrm{C}$, enquanto na simulação a temperatura encontrada foi de $693^{\circ} \mathrm{C}$, ou seja, um erro de $5,6 \%$, conforme Equação 8. Este desvio pode ser corrigido com o ajuste da emissividade dos gases da combustão.

$$
\text { erro }=\frac{\left(T_{\text {experimental }}-T_{\text {simulaçäo }}\right)}{T_{\text {experimental }}} \cdot 100 \%
$$




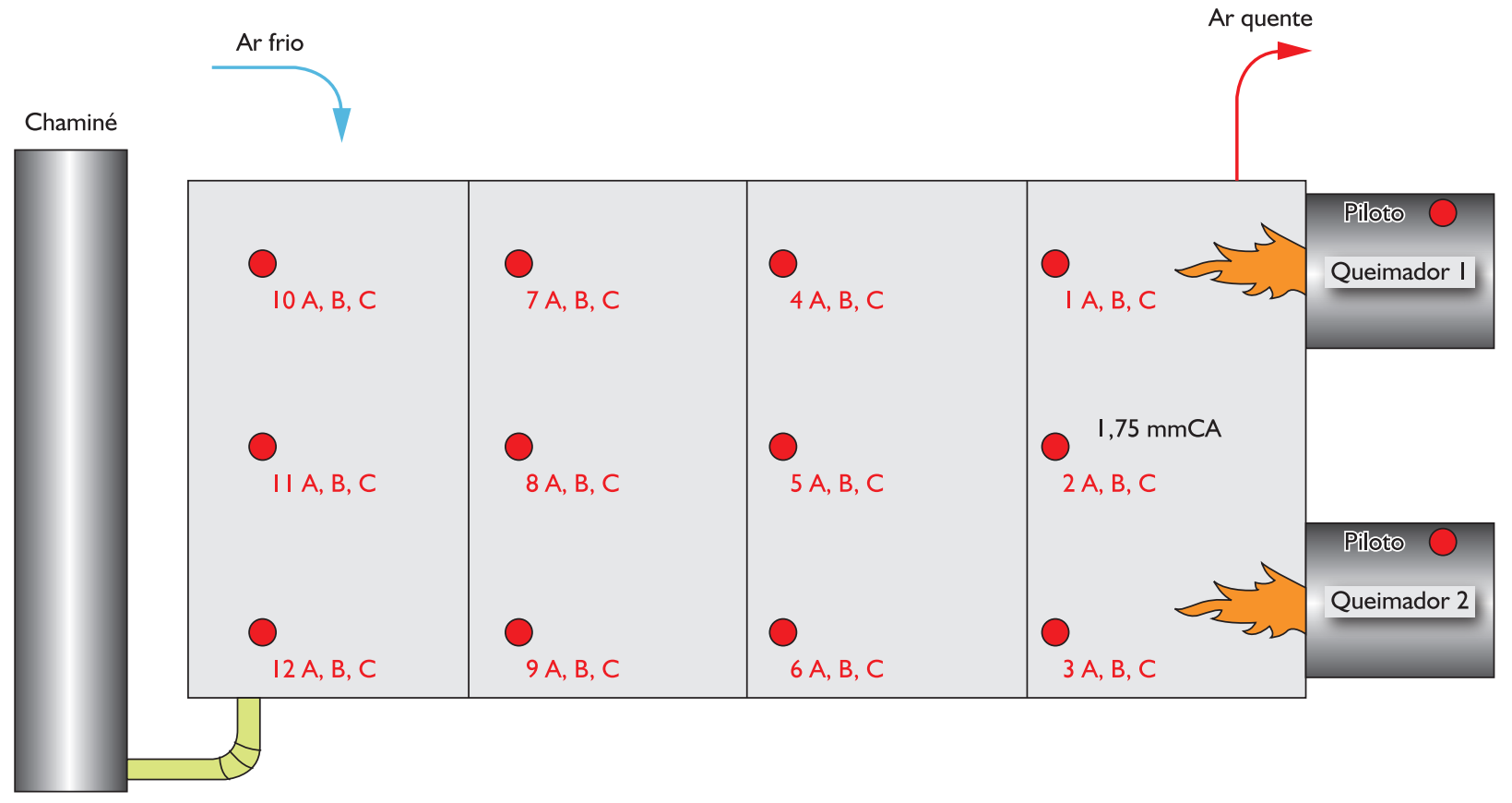

Figura 4. Vista superior dos pontos de medição. $^{(7)}$

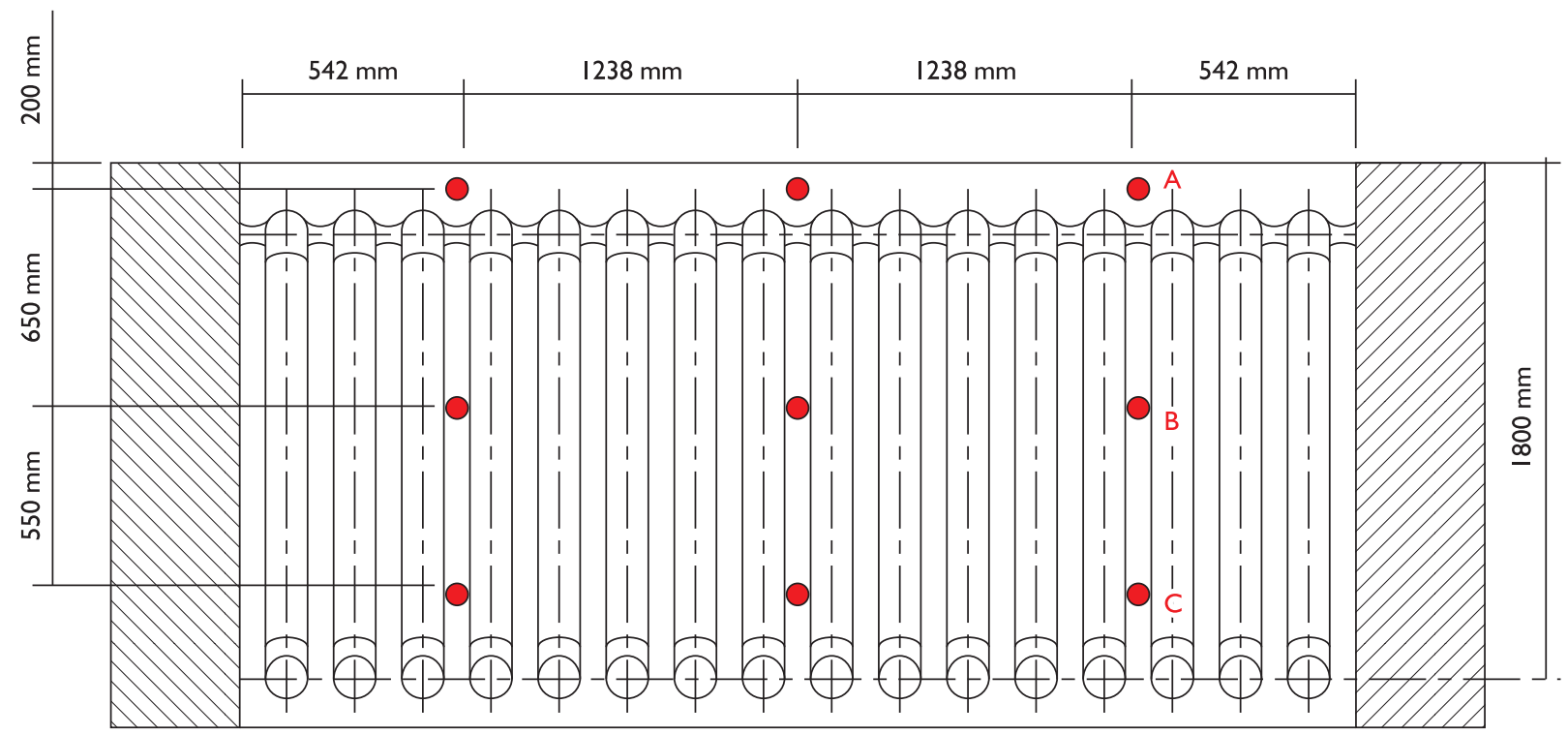

Figura 5. Posição dos instrumentos de medição na seção transversal do Glendon. ${ }^{(7)}$

A Tabela I mostra os resultados do balanço de energia para as três simulações.

É possível observar que para as mesmas condições de entrada (energia dos queimadores e entrada de ar nas serpentinas) existe um gradativo aumento da energia que é transferida dos gases da combustão para o ar no interior das serpentinas.

Desta forma, a eficiência energética dos Glendons que é $59,1 \%$ passaria para $67,4 \%$ conforme Equação 9.

$$
\begin{aligned}
& \eta=\frac{\dot{\mathrm{Q}}_{\text {util }}}{\dot{\mathrm{Q}}_{\text {total }}}=\frac{\dot{\mathrm{Q}}_{\mathrm{ar}}}{\dot{\mathrm{Q}}_{\text {gases }}}= \\
& =\frac{(\text { Potência saída do ar })-(\text { Potência de entrada do ar })}{\text { Potência dos queimadores }}
\end{aligned}
$$

O aumento da eficiência energética pode ser atribuído a dois fatores, o aumento da turbulência dos gases e a alteração do escoamento dos gases da combustão, que passou de um escoamento paralelo para um escoamento cruzado em relação ao escoamento de ar. 
Tabela I. Resultado dos balanços energéticos

\begin{tabular}{lccc}
\hline \multicolumn{1}{c}{ Posição } & Etapa I & Etapa 2 & Etapa 3 \\
\hline Queimadores (MW) & 3,67 & 3,67 & 3,67 \\
Entrada de ar nas serpentinas (MW) & 0,232 & 0,232 & 0,232 \\
Saída dos gases da combustão (MW) & 1,5 & 1,434 & $\mathrm{I}, 18 \mathrm{I}$ \\
Saída do ar nas serpentinas (MW) & 2,4 & 2,464 & 2,706 \\
\hline
\end{tabular}

O aumento na eficiência energética é evidenciado no aumento da temperatura de saída do ar nas serpentinas, conforme Tabela 2.

As Figuras 6 e 7 mostram a evolução do perfil de temperatura ao longo do Glendon e em uma seção transversal, nas 3 etapas de simulação.

Tabela 2. Resultados de temperatura do ar de saída e dos gases na chaminé

\begin{tabular}{lccc}
\hline \multicolumn{1}{c}{ Posição } & Etapa I & Etapa 2 & Etapa 3 \\
\hline Saída do ar nas serpentinas $\left({ }^{\circ} \mathrm{C}\right)$ & 693 & 710 & 808 \\
Saída dos gases da combustão $\left({ }^{\circ} \mathrm{C}\right)$ & 381 & 378 & 317 \\
\hline
\end{tabular}

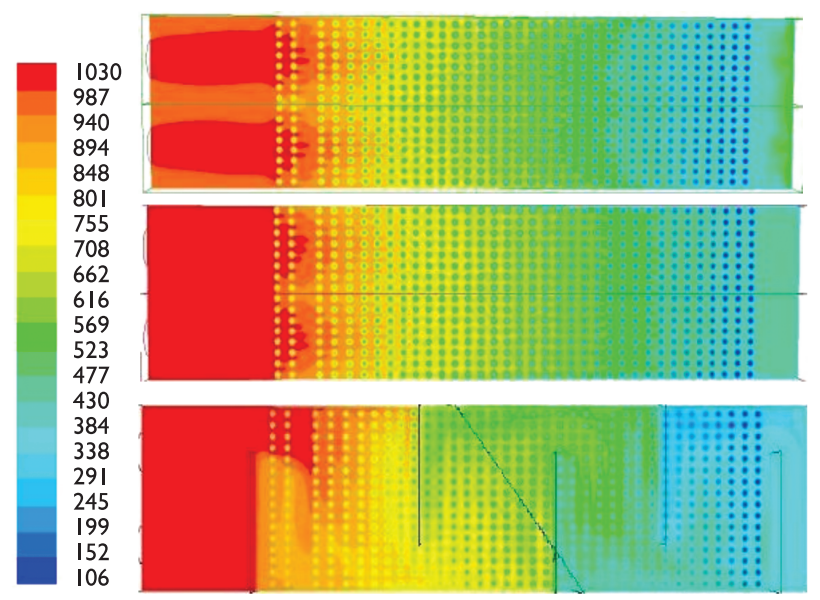

Figura 6. Evolução das temperaturas $\left({ }^{\circ} \mathrm{C}\right)$ - vista da planta. ${ }^{(11)}$

A configuração do Glendon na primeira simulação apresenta duas regiões de alta temperatura dos gases da combustão em frente a cada queimador. As temperaturas de saída dos gases de combustão são maiores nesta simulação se comparadas com as temperaturas das simulações seguintes, isto se deve à menor eficiência de troca térmica entre os fluidos.

Na seção transversal, é possível notar que a temperatura dos gases da combustão é mais elevada na região superior, pois, devido à preferência de escoamento dos gases da combustão por esta região, os gases não trocam calor com $\circ$ ar. A distância entre $\circ$ topo das serpentinas e o teto é de $200 \mathrm{~mm}$.

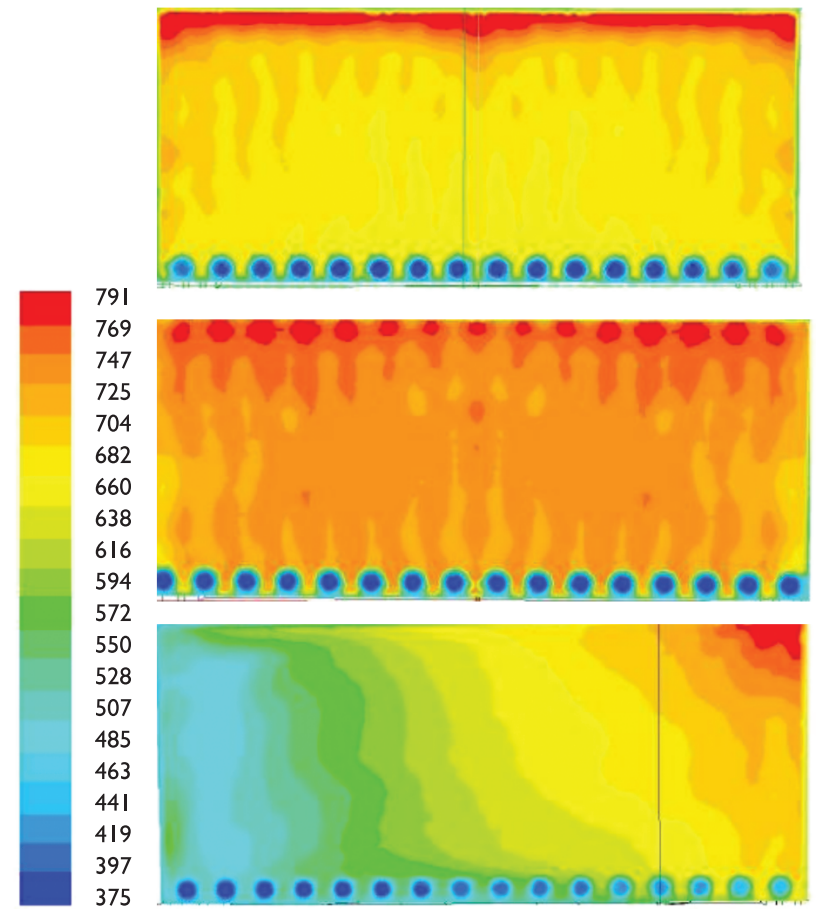

Figura 7. Evolução das temperaturas $\left({ }^{\circ} \mathrm{C}\right)$ - seção transversal..$^{(11)}$

A partir da verificação que a temperatura dos gases da combustão é mais elevada na parte superior do Glendon, foram modelados na segunda simulação o rebaixamento do teto e a aproximação das paredes em relação às serpentinas. É possível perceber uma maior homogeneização da temperatura na câmara de combustão devido ao aumento da turbulência nesta região. Mas, pouca diferença pode ser percebida ao longo de todo o Glendon.

$\mathrm{Na}$ segunda simulação, o perfil de temperatura na seção transversal foi alterado sensivelmente. A região superior de escoamento preferencial dos gases foi reduzida e a distância simulada entre o topo das serpentinas e o teto é de $50 \mathrm{~mm}$.

$\mathrm{Na}$ terceira simulação, foi modelada a inserção de cinco barreiras, que funcionam como chicanas. Estas barreiras alteraram o escoamento dos gases da combustão, que passou de paralelo para cruzado. A Figura 6 mostra que o gradiente de temperatura na vista de planta é predominantemente longitudinal, na primeira e na segunda simulação. $\mathrm{Na}$ terceira simulação, o gradiente de temperatura não possui uma única direção. As chicanas promoveram uma troca de calor mais eficiente, e isso pode ser observado pela diminuição dos valores de temperatura dos gases na saída do Glendons.

O perfil de temperatura na seção transversal também sofreu alteração. $O$ gradiente de temperatura mais significativo está em uma direção oblíqua, e não mais na direção vertical, como nas simulações anteriores. Apesar de algumas regiões "frias" numa mesma seção transversal, o ganho global de temperatura do ar na saída dos Glendons na terceira simulação foi de $115^{\circ} \mathrm{C}$. 
Com base nos resultados do modelo computacional, as barreiras que funcionam como chicanas foram montadas em um dos três Glendons e outras melhorias foram executadas, como substituição das válvulas de controle de vazão de gás, substituição dos queimadores de chama longa por queimadores de chama curta e substituição da válvula de controle de pressão de gás combustível. $\mathrm{O}$ aumento real de temperatura do ar na saída dos Glendons após as modificações foi de $135^{\circ} \mathrm{C}$, não podendo ser maior devido a limitações de outras partes do equipamento. A temperatura da câmara de combustão se manteve abaixo dos $1.050^{\circ} \mathrm{C}$, valor máximo estabelecido, enquanto a temperatura do ar de saída alcançou valores próximos a $870^{\circ} \mathrm{C}$, conforme mostrado na Figura 8 . Estes dados foram obtidos a partir das medições realizadas pelos instrumentos do próprio Glendon.

Para relacionar o aumento da temperatura do ar na saída dos Glendons com a redução potencial do consumo de oxigênio, a Equação 10 foi utilizada, pois esta é a equação usada na operação dos altos-fornos da V\&M do Brasil para calcular a temperatura de chama.

$$
T_{\text {chama }}=1463+0,7 . T_{\text {arsopro }}+44 O_{2}-. U_{\text {ar }}-1,5 I_{\text {finos }}
$$

onde, $\mathrm{T}_{\text {arsopro }}$ é a temperatura do ar de sopro, $\mathrm{O}_{2}$ é a porcentagem de enriquecimento do ar de sopro com oxigênio, $U_{a r}$ é a umidade relativa do ar e $I_{\text {finos }}$ é a taxa de injeção de finos de carvão.

Considerando a vazão de ar do alto-forno e os parâmetros. $\mathrm{T}_{\text {chama }}, \mathrm{U}_{\text {ar }}$ e $\mathrm{I}_{\text {finos }}$ constantes, foi possível

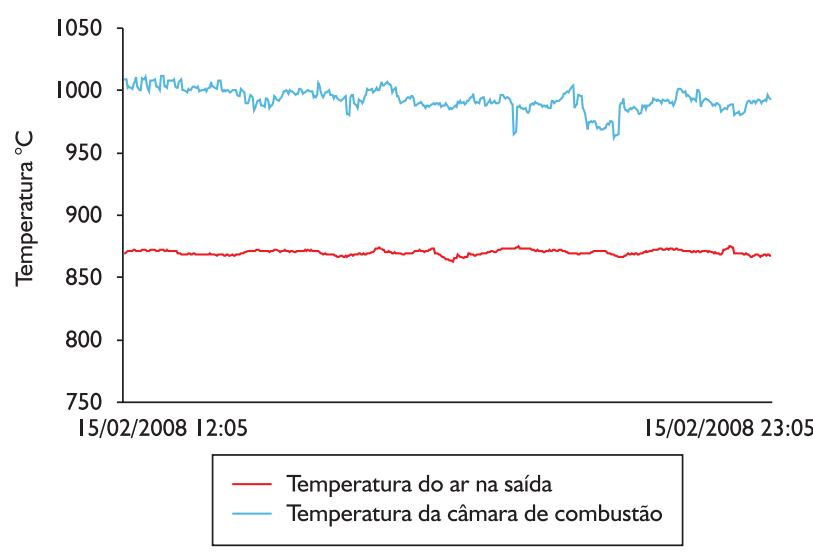

Figura 8. Temperatura do ar na saída e temperatura da câmara de combustão. ${ }^{(12)}$ construir a Figura 9 que mostra que, para o aumento de temperatura do ar de sopro de $115^{\circ} \mathrm{C}$ previsto pelo modelo, seria possível obter uma redução do consumo anual de oxigênio da ordem de $4.400 .000 \mathrm{Nm}^{3}$.

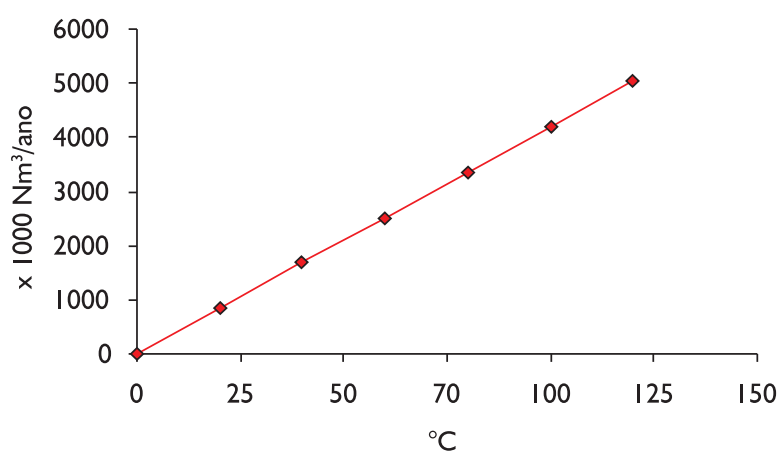

Figura 9. Economia anual de oxigênio em função do aumento de temperatura do ar de sopro. ${ }^{(13)}$

\section{CONCLUSÃO}

O modelo computacional se mostrou uma ferramenta importante para o entendimento do comportamento fluidodinâmico dos Glendons da V\&M do Brasil. A partir deste modelo foi possível simular duas modificações geométricas no equipamento objetivando o aumento de sua eficiência energética e vida útil.

A primeira alteração consistiu em reduzir a área de passagem dos gases da combustão através do rebaixamento do teto e da aproximação das paredes em relação às serpentinas. A segunda alteração consistiu na inclusão de cinco barreiras (chicanas) que bloqueiam dois terços da área da seção transversal dos Glendons.

A inclusão das chicanas se mostrou mais efetiva do que a redução da área de passagem dos gases da combustão, e promoveu um aumento na eficiência energética do Glendons de $59,1 \%$ para $67,4 \%$.

Em termos de temperatura, o modelo computacional previu um aumento de $115^{\circ} \mathrm{C}$ na saída do ar das serpentinas, mantendo-se as mesmas condições de entrada. No teste experimental este aumento de temperatura chegou a $135^{\circ} \mathrm{C}$. Considerando o aumento teórico de $115^{\circ} \mathrm{C}$, a redução do consumo de oxigênio no Alto-Forno 2 será de aproximadamente $4.400 .000 \mathrm{Nm}^{3}$ por ano, mantendo a mesma temperatura de chama no Alto-Forno.

\section{REFERÊNCIAS}

I KOVAL, I. F.; KRAVCHENKO, A. Y. Continuous temperature control in the combustion zone of a blast furnace. Metallurgist, v. I, n. 3, p. I45-8, 1958.

2 ROSTOVISKII, A.V.; PARENKOV, A. E.; CHERNOUSOV, P. I. Refining theoretical combustion temperature during the blow-in of a blast furnace. Metallurgist, v. 42, n. 12, p. 474-6, Dec. 1998. 
3 GASIN. Técnicas oxigênio-combustível. Disponível em: <http://www.gasin.pt/htm/your_business/Glass_OxyFuelTech. htm >. Acesso em: I jun. 2006.

4 CASTRO, L. F. A. Melhorias operacionais em altos-fornos com stave-cooler. Belo Horizonte: Vallourec \& Mannesmann do Brasil, 2005.

5 LALOVIC, M.; RADOVIC, Z.; JAUKOVI, N. Flame temperature as a function of the combustion conditions of gaseous fuels. Materiali in Tehnologije, v. 40, n. 3, p. 89-92, Apr. 2006.

6 ASSUNÇÃO, C. S. Simulação computacional do comportamento termodinâmico de um Glendon. In: CONGRESSO ANUAL DA ABM, 62., 2007, Vitória. Anais... São Paulo: ABM, 2007. p. I64I-50.

7 ASSUNÇÃO, C. S. Glendon siderúrgico: análise termodinâmica e modelagem matemática simplificada. 2006. II5 f. Dissertação (Mestrado em Engenharia Mecânica) - Departamento de Engenharia Mecânica da Universidade Federal de Minas Gerais, Belo Horizonte, 2006.

8 VERSTEEG, H.; MALALASEKERA, W. An introduction to computational fluid dynamics. New York: Longman, 1995.

9 ASSUNÇÃO, C. S. et al. Blast furnace air pré-heater. Ansys Advantage, v. I, n. I, p. 29, 2007.

10 ASSUNÇÃO, C. S. Reduction of oxygen consumption in a blast furnace through the improvement of heat exchangers efficiency. In: ASME INTERNATIONAL MECHANICAL ENGINEERING CONGRESS AND EXPOSITION, 2008, Boston. Proceedings... New York: ASME, 2008. IMECE2008-68559.

II ASSUNÇÃO, C. S. Simulação computacional do comportamento termodinâmico de um Glendon. In: SEMINÁRIO DE REDUÇÃO DE MINÉRIO DE FERRO E MATÉRIAS-PRIMAS, 37., 2007, Salvador. Anais... São Paulo: ABM, 2007. p. 437-47.

I2 VALLOUREC \& MANNESMAN DO BRASIL. PIMS - Process Information Management System. Belo Horizonte, 2008. [Relatório]

13 ASSUNÇÃO, C. S. Redução do consumo de oxigênio em um alto-forno a partir do aumento da eficiência energética dos trocadores de calor. In: ENCONTRO DE PRODUTORES E CONSUMIDORES DE GASES INDUSTRIAIS, 25. SEMINÁRIO DE BALANÇOS ENERGÉTICOS GLOBAIS E UTILIDADES, 30., 2009, São Paulo. Anais.. São Paulo: $A B M$, 2009. p. 85-94.

Recebido em: 19/10/2006

Aceito em: $31 / 03 / 201 \mathrm{I}$ 